\title{
Biological artificial valve dysfunction - single-centre, observational echocardiographic study in patients operated on before age 65 years
}

\author{
Marek Maciejewski ${ }^{1}$, Katarzyna Piestrzeniewicz ${ }^{1}$, Agata Bielecka-Dabrowa², Andrzej Walczak ${ }^{3}$
}

1Department of Cardiology, Institute of Cardiology and Cardiac Surgery, Medical University of Lodz, Poland

2Department of Hypertension, Medical University of Lodz, Poland

${ }^{3}$ Department of Cardiac Surgery, Institute of Cardiology and Cardiac Surgery,

Medical University of Lodz, Poland

Submitted: 24 January 2011

Accepted: 9 June 2011

Arch Med Sci 2011; 7, 6: 993-999

DOI: 10.5114/AOMS.2011.26611

Copyright @ 2011 Termedia \& Banach

\section{Abstract}

Introduction: Patients with implanted bioprostheses are at risk of structural dysfunction which results from the limited durability of biological valves. The aim of this study was to analyse the mechanism of bioprosthesis degeneration and to evaluate the usability of transthoracic and transoesophageal echocardiography in determining the indications for reoperation in 117 patients with a bioprosthesis implanted before 65 years old.

Material and methods: The study comprised 117 consecutive patients ( $M-27$, $\mathrm{F}-90$, age $48-74$ years, $57.5 \pm 9.5$ years) with a bioprosthesis implanted under the age of 65 , who were examined in accordance with the accepted protocol and whose complete clinical and echocardiographic documentation was collected. The scheduled echocardiographic examination was performed annually from the 5 year after implantation of the bioprosthesis in patients with a valve implanted over the age of 35 years and from the 1 year after bioprosthesis implantation in patients with a prosthesis implanted at a younger age. Unscheduled echocardiographic examinations were performed only on clinical indications.

Results: During the period under observation, due to degeneration of the bioprosthesis 76 patients were reoperated, including 62 patients with mitral bioprostheses. In $88.7 \%$ of patients with degeneration of mitral valve bioprostheses, regurgitation was observed. In $69 \%$ of patients with aortic bioprostheses, valve dysfunction was the dominant mechanism of stenosis.

Conclusions: The most common mechanism of structural dysfunction of a mitral bioprosthesis is regurgitation caused by prolapse or perforation of one of the leaflets. Degeneration of an aortic bioprosthesis usually results in aortic stenosis. In cases of bioprosthesis degeneration connected with stenosis, transthoracic echocardiography was sufficient for the evaluation of valve dysfunction. In the case of bioprosthesis dysfunction accompanied by regurgitation, transoesophageal echocardiography was more informative to decide when the operation should be performed.

Key words: bioprosthesis, transthoracic echocardiography, transoesophageal echocardiography, reoperation.

\section{Introduction}

Patients with implanted bioprostheses are at risk of structural dysfunction which results from the limited durability of biological valves. Dura-

\author{
Corresponding author: \\ Marek Maciejewski MD, PhD \\ Clinic of Cardiology \\ Institute of Cardiology \\ and Cardiac Surgery \\ Medical University of Lodz \\ 1/3 Sterlinga St \\ 91-425 Lodz, Poland \\ Phone/fax: +48 426364471 \\ E-mail: marek56maciejewski@ \\ gmail.com
}


bility of this type of valve is dependent mainly on the patient's age at the time of prosthesis implantation and its localization [1-3]. Clinical signs of artificial biological prosthetic valve dysfunction, resulting both from its damage and complications not related to the valve itself, are similar. Diagnosis is often possible after echocardiography, usually transoesophageal, is performed. Structural dysfunction of a bioprosthesis implanted in patients after 65 years of age, according to the majority of authors, results from its mineralization and usually develops in the form of valve stenosis [4-6]. Our own observations of a group of patients under the age of 65 years with implanted bioprostheses indicate a different mechanism of degeneration occurring in these subjects.

Table I. Characteristics of the 117 patients with implanted bioprostheses

\begin{tabular}{|c|c|c|}
\hline Age [years] & $\begin{array}{c}\text { Mean } \\
\text { (from-to) }\end{array}$ & $\begin{array}{c}57.5 \pm 9.6 \\
(48-74)\end{array}$ \\
\hline Sex & $M(\%) / F^{*}$ & $27(23) / 90$ \\
\hline $\begin{array}{l}\text { Mitral } \\
\text { commissurotomy }\end{array}$ & $n(\%)$ & $38(32.5)$ \\
\hline $\begin{array}{l}\text { Age during valve } \\
\text { replacement [years] }\end{array}$ & $\begin{array}{c}\text { Mean } \\
\text { (from-to) }\end{array}$ & $\begin{array}{c}47.3 \pm 9.6 \\
(37-65)\end{array}$ \\
\hline \multicolumn{3}{|l|}{ Type of bioprosthesis: } \\
\hline One mitral & $n$ & 79 \\
\hline One aortic & $n$ & 23 \\
\hline One tricuspid & $n$ & 2 \\
\hline Mitral and aortic & $n$ & 13 \\
\hline Total & $n$ & 130 \\
\hline $\begin{array}{l}\text { Time from prosthetic } \\
\text { valve implantation } \\
\text { [months] }\end{array}$ & $\begin{array}{c}\text { Mean } \\
\text { (from-to) }\end{array}$ & $\begin{array}{c}121.5 \pm 4.0 \\
(13-230)\end{array}$ \\
\hline Atrial fibrillation & $n(\%)$ & $79(67.5)$ \\
\hline Embolic complications & $n(\%)$ & $8(6.8)$ \\
\hline Reoperations & $n(\%)$ & $89(76.0)$ \\
\hline Structural dysfunction & $n(\%)$ & $76(65.0)$ \\
\hline Infective endocarditis & $n(\%)$ & $13(11.0)$ \\
\hline
\end{tabular}

The aim of this study was to analyse the mechanism of bioprosthesis degeneration and to evaluate the usability of thoracic and transoesophageal echocardiography in the monitoring of the biological valve degeneration process.

\section{Material and methods}

The study comprised 117 consecutive patients with a bioprosthesis implanted under the age of 65, who were examined between 1987 and 2009 in accordance with the accepted protocol [7] (Table I), and whose complete clinical and echocardiographic documentation was collected. In the study group there were 27 men and 90 women. Mitral valves were implanted in 79 patients, aortic valves in 23 patients, tricuspid valves in 2 patients and two artificial valves in 13 patients. A total of 130 bioprostheses were implanted, including 122 bioprostheses from Carpentier-Edwards and 8 from other manufacturers. The age of patients at the end of observation ranged from 48 to 74 years (mean 57.5 \pm 9.5 years). Twenty-three patients were in NYHA functional class III at that time, the others in NYHA functional class I-II. During the follow-up period, 76 patients underwent a valve replacement because of its degeneration, and 13 patients were reoperated because of infective endocarditis. Information concerning the types of biological valves which required exchange is shown in Table II. In order to fulfil the aim of this study, 13 patients with infective endocarditis were excluded from further analysis, and thus 104 patients, who did not differ demographically from the initially presented group of patients with implanted bioprostheses, were subjected to a final evaluation.

\section{Study protocol according to ESC guidelines}

The scheduled echocardiographic examination was performed annually from the $5^{\text {th }}$ year after implantation of the bioprosthesis in patients with a valve implanted over the age of 35 years and from the $1^{\text {st }}$ year after bioprosthesis implantation in patients with a prosthesis implanted at a younger age. Unscheduled echocardiographic examinations were performed only on clinical

Table II. Types of bioprostheses and types of their dysfunction in 89 reoperated patients

\begin{tabular}{|c|c|c|c|c|c|c|}
\hline Type of dysfunction & & $\mathrm{pC}-\mathrm{E}$ & periC-E & I-S & LIOTTA & Total \\
\hline Infective endocarditis & $n(\%)$ & 12 & - & - & 1 & $13(14.6)$ \\
\hline Bioprosthesis structural degeneration & $n(\%)$ & 65 & 4 & 2 & 5 & $76(85.4)$ \\
\hline Stenosis & $n(\%)$ & 14 & 1 & - & 2 & $17(22.4)^{\star}$ \\
\hline Regurgitation & $n(\%)$ & 51 & 3 & 2 & 3 & $59(77.6)^{\star}$ \\
\hline Together & & 77 & 4 & 2 & 6 & 89 \\
\hline
\end{tabular}

pC-E-porcine Carpentier-Edwards bioprosthesis, periC-E - pericardial Carpentier-Edwards bioprosthesis, I-S - Ionescu-Shiley, ${ }^{*}$ percentage applies to patients with structural bioprosthesis degeneration 
indications. All standard transthoracic and transoesophageal projections were initially recorded on Super-VHS videotape, and from 1998 on a digital carrier. Bioprosthesis degeneration was diagnosed when thickening of cusps with reduction of their mobility and with an increase in the flow speed through the valve or the occurrence or increasing severity of valvular regurgitation were observed [7].

During the transoesophageal examination particular attention was paid to disturbances in bioprosthesis cusp mobility, especially to their prolapse or the presence of perforation. The mechanism of bioprosthesis degeneration was evaluated on the basis of transoesophageal examination performed in 5 patients with an urgent indication and in the remaining 99 patients because of abnormalities suspicion in TTE. During this examination, the frequency of thrombotic material occurrence in the heart and the thoracic aorta and the presence of perivalvular leak were also evaluated. The usability of transthoracic and transoesophageal echocardiography in the recognition of bioprosthesis structural dysfunction and its mechanism was evaluated on the basis of the intraoperative image. The analysis of consecutive echocardiograms of patients allowed for the evaluation of the progress of bioprosthesis structural dysfunction depending on its mechanism [7].

\section{Results}

The first, scheduled echocardiographic examination performed in patients with normal, according to clinical assessment, function of the artificial valve demonstrated the presence of insignificant structural regurgitation in 35 patients (30\%) and suggested thickening of the valve leaflets in 12 patients. Until the completion of studies, on average, 121.5 months after bioprosthesis implantation, 76 patients were reoperated. The remaining 28 patients were under clinical and echocardiographic monitoring. The results presented below apply to preoperative transthoracic and transoesophageal echocardiograms of reoperated patients and echocardiograms made in the last year of observation of patients without valve replacement.

\section{Transthoracic study}

Morphological assessment of bioprosthesis leaflets showed their thickening in 32 patients and in 32 patients prolapse or perforation of the biological valve was observed. In the remaining 40 patients the morphology of leaflets was assessed as normal. Functional assessment showed significant valve stenosis in 19 patients, its significant regurgitation in 24 patients and normal function of the valvular prosthesis in the remaining 61 patients (Figure 1).

\section{TTE, $n=104$}
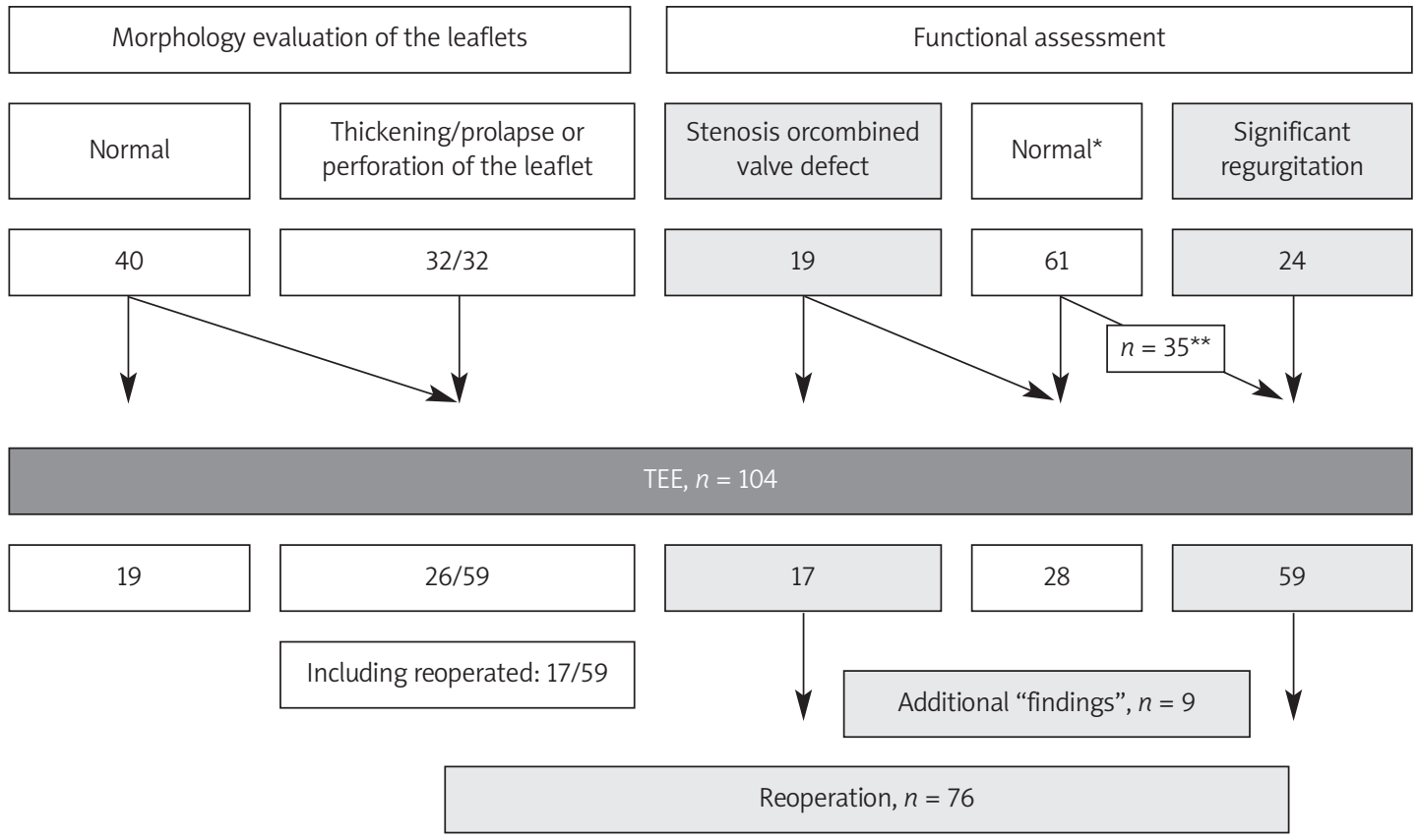

Figure 1. Results of transthoracic (TTE) and transoesophageal (TEE) echocardiography in 104 patients with implanted bioprostheses

*Also non-significant regurgitation or stenosis, ** patients with significant mitral regurgitation assessed in transthoracic echocardiog raphy as non-significant 

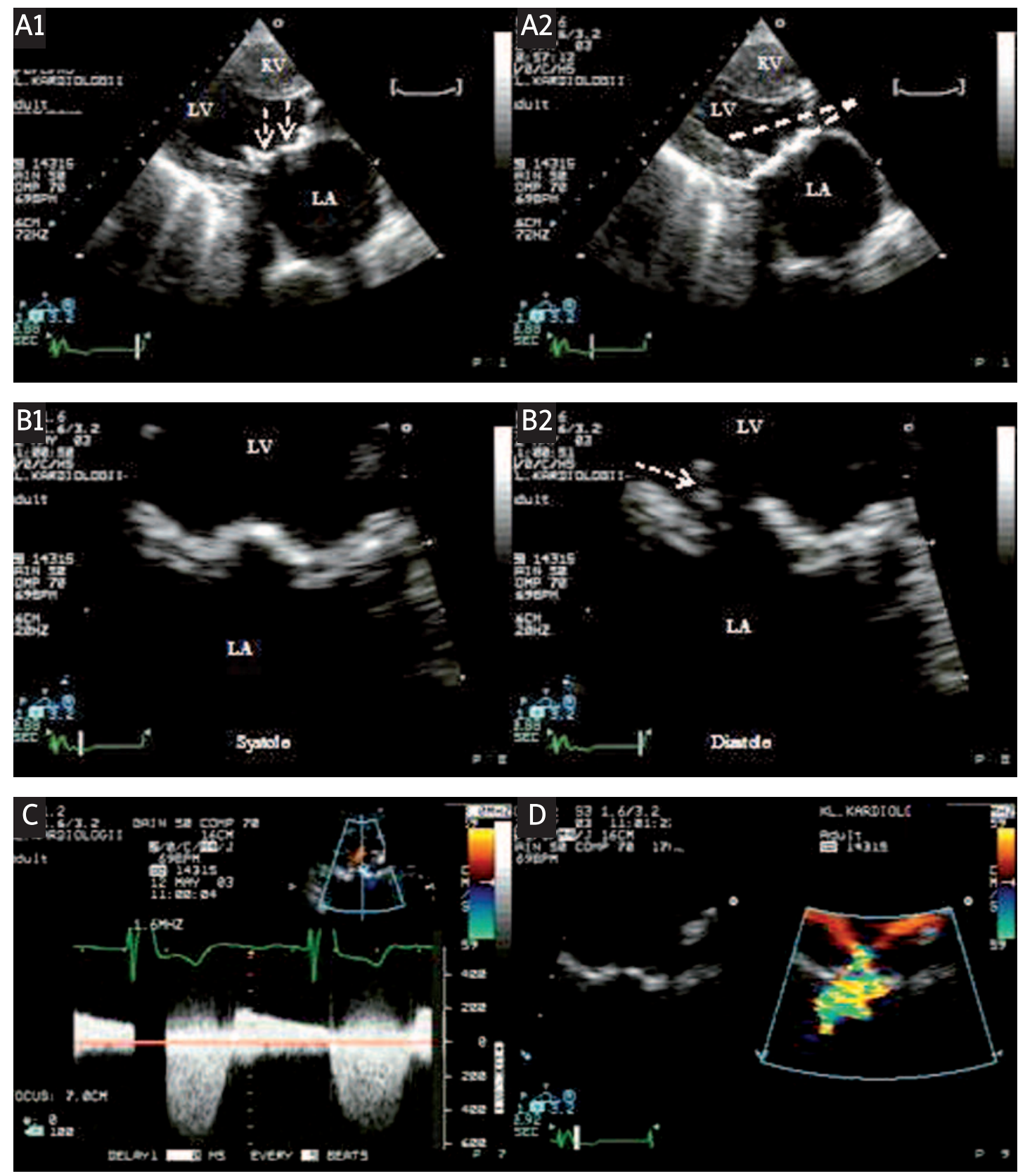

Figure 2. Patient, 63 years old, male, class III NYHA. Mitral pericardial Carpentier-Edwards bioprosthesis implanted 12 years ago. Transthoracic echocardiography: A1 - parasternal long axis view: extensive calcification of the valve leaflets with a significant reduction of their mobility (arrows). A2 - parasternal long axis view: improper angle between the axis of the valve and left ventricular outflow tract. B1 and B2 - apical 4-chamber view: maintained sufficient mobility of one of the leaflets (arrow). C and D - apical 4-chamber view (continuous wave Doppler echocardiography): haemodynamically significant combined mitral valve defect

\section{Transoesophageal study}

Morphological assessment of bioprosthesis leaflets demonstrated their thickening in $26 \mathrm{pa}$ tients. Thickening of leaflets observed during transthoracic examination was not confirmed in the remaining 6 patients. Prolapse or perforation of bioprosthesis leaflets was reported in a total of 59 patients, including 32 patients with the diagnosis made on the basis of transthoracic examination.
No morphological changes of leaflets were seen in 19 patients (Figure 1 ).

Functional assessment confirmed the presence of stenosis in 17 out of 19 patients with such a diagnosis made on the basis of transthoracic examination (Figure 2) and revealed the presence of significant regurgitation in 59 patients (Figure 3), including 24 subjects with such a diagnosis made on the basis of transthoracic examination. In the remaining 28 patients bioprosthesis function was 

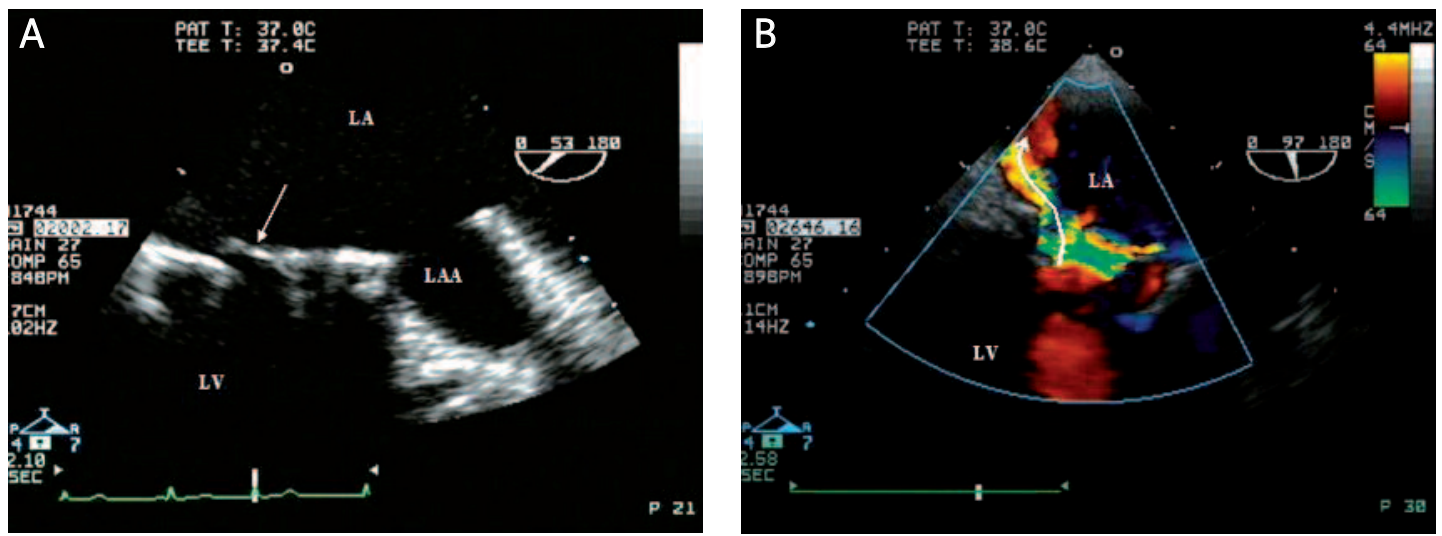

Figure 3. Patient, 67 years old, male, class III NYHA. Mitral porcine Carpentier-Edwards bioprosthesis implanted 13 years ago. Transoesophageal echocardiography: A - mid-oesophageal view $53^{\circ}$ - bioprosthetic valve, B - mid-oesophageal view 97 degrees (colour flow Doppler) - multidirectional mitral regurgitation connected with valve prolapse

assessed as normal. Moreover, during the transoesophageal examination, potential embolic sources were found in 9 patients (8.6\%), including 6 patients with thrombotic material in a single location and 3 patients with multiple embolic sources. In 3 patients, thrombus was found on the ring of the bioprosthesis, in 5 patients it was present in the left atrium or its auricle, and in 4 patients complex atherosclerotic plaques in the thoracic aorta were reported. The presence of spontaneous echo contrast was observed in the left atrium of 55 patients, and in the aorta of 2 patients. Haemodynamically insignificant paravalvular leak was found in 5 patients.

\section{Indications for reoperation}

Seventy-six patients were qualified for reoperation. In all patients intraoperative assessment confirmed the diagnosis made on the basis of transoesophageal examination. Valve regurgitation resulting from prolapse of the leaflet or its perforation was the reason for reoperation in 59 patients $(77.6 \%)$, while stenosis or stenosis with valve regurgitation was the reason in the remaining 17 patients. Among 62 patients reoperated due to structural dysfunction of a mitral bioprosthesis, dominant regurgitation was seen in 55 patients (88.7\%). Among 13 patients with an aortic bioprosthesis, stenosis was the predominant mechanism of dysfunction (9 patients, $69 \%$ ).

\section{The choice of reoperation date}

The analysis of the progression of bioprosthesis structural dysfunction revealed rapid acceleration of this process in cases when regurgitation was the dominant mechanism of degeneration. Forty-five patients (76.3\%) with bioprosthesis regurgitation reported for the first time or with worsening of regurgitation required reoperation during the first year, usually during the first 6 months, since the first detection of a dicrotic wave. Worsening of regurgitation or the appearance of clinical symptoms (increase in the degree of heart failure) was the direct reason for reoperation. Five patients were reoperated for urgent indications. If any feature of valvular stenosis was observed, the progression of structural dysfunction was usually slow. Ten out of 13 patients with stenosis as the predominant mechanism of degeneration required reoperation $\geq 2$ years after the first detection of features of structural dysfunction.

\section{Discussion}

Structural dysfunction of the bioprosthesis was the most common reason for reoperation in our study (76 patients $-39.2 \%$ ). Also, other authors indicate that bioprosthesis degeneration is the most frequent reason for valvular reoperation [8-10]. The necessity of reoperation of every patient whose survival is long results from the limited durability of the bioprosthesis [11], especially in patients operated on under the age of 60-65 years [10, 12]. Good long-term results of bioprosthesis implantation in older patients are well known [13-15]. Long-term results of implantation of currently available bioprostheses are comparable [16, 17]. Very good results of bioprosthesis implantation in patients who underwent surgery under the age of 60 years and were followed for over 20 years were presented by Ruel et al. [18].

This study comprises only patients with bioprostheses implanted under the age of 65 years. Average durability of bioprostheses was about 10 years. These observations do not differ from earlier reports [19, 20]. Van Doorn et al. [21] reported rapid increase in the frequency of degeneration of the standard Carpentier-Edwards bioprosthesis from the $7^{\text {th }}$ year after its implantation. Higher durability of some bioprosthesis models was described 
in several reports $[12,18]$. The impact of the small size of the aortic bioprosthesis on its earlier degeneration is also known [22].

In our study, valvular regurgitation was a direct indication for reoperation in most patients (77.6\%) and mitral valve regurgitation was the dominant mechanism of dysfunction in $88.7 \%$ of patients. Other authors reported that regurgitation of the mitral bioprosthesis was the dominant mechanism of dysfunction only in $30 \%$ of patients $[23,24]$. These differences may result from the demographic dissimilarity of study group of patients as well as from the rheumatic aetiology of operated heart defects presented in this paper. Perennial course of mitral stenosis and significant enlargement of the left atrium resulted in that the axis of the implanted bioprosthesis usually was not perpendicular to the axis of the left ventricle (Figure 2). This could lead to a local change of haemodynamic conditions and functions of particular bioprosthesis leaflets. The most common indication for reoperation of patients with implanted aortic valves was stenosis or complex defect with a predominant stenosis, which is consistent with previous reports $[25,26]$.

The usability of transthoracic echocardiography in the diagnosis and in the assessment of the severity of bioprosthesis structural dysfunction was limited. In our study, morphological assessment of leaflets was demonstrated to over-recognize the thickening of bioprosthesis leaflets but, on the other hand, it failed to detect bioprosthesis prolapse in some patients. In the functional assessment, transthoracic echocardiography was sufficient for the identification and evaluation of bioprosthesis stenosis degree; however, specific assessment of the degree of mitral valve regurgitation by this method was possible only in $41 \%$ of patients. In the remaining patients, the dicrotic wave through the bioprosthesis was visualized during transthoracic examination, but it was assessed as irrelevant. These patients derived the greatest benefit from the transoesophageal examination. These observations indicated serious limitations of transthoracic echocardiography in the diagnosis of bioprosthesis structural dysfunction accompanied by regurgitation due to leaflet prolapse.

In patients with detected potential sources of systemic embolism, atrial fibrillation, symptoms of heart failure (mainly NYHA functional class III) and advanced age (usually over 65 years) were more frequent.

Bioprosthesis structural dysfunction does not lead to the increase in perioperative risk, if reoperation is carried out as scheduled $[8,12,18]$. In this study, only 5 patients were reoperated for urgent indications, all due to a sharp worsening of circulatory function resulting from mitral bioprosthesis regurgitation. Rapid deterioration in clinical state of patients with bioprosthesis degeneration accompanied by its regurgitation can be explained by the type of valve leaflet damage. Perforation or leaflet prolapse often accompanied by its "flail" movement results in hemodynamic consequences comparable to those of acute mitral regurgitation. The remaining 71 patients were reoperated according to schedule. These patients were monitored every 3-6 months during the final year before the reoperation. The percentage of patients reoperated according to the schedule did not differ from that reported by other authors [11, 12, 18, 21].

In conclusion, the most common mechanism of structural dysfunction of a mitral bioprosthesis is regurgitation caused by prolapse or perforation of one of the leaflets. Degeneration of an aortic bioprosthesis usually results in aortic stenosis. Transthoracic echocardiography is sufficient for the periodic monitoring of patients with implanted bioprostheses. Transoesophageal echocardiography is more accurate for patients with mitral bioprosthesis dysfunction accompanied by regurgitation. There is a group of patients aged 65 years with implanted bioprostheses, chronic atrial fibrillation and chronic heart failure, in whom transoesophageal echocardiography can give relevant information regarding the left atrial auricle and thoracic aorta, in the context of the scheduled reoperation.

\section{References}

1. Jamieson WR, Rosado LJ, Munro Al, et al. CarpentierEdwards standard porcine bioprosthesis: primary tissue failure (structural valve deterioration) by age groups. Ann Thorac Surg 1988; 46: 155-62.

2. Burr LH, Jamieson WR, Munro Al, et al. Structural valve deterioration in elderly patient populations with the Carpentier-Edwards standard and supra-annular porcine bioprostheses: a comparative study. J Heart Valve Dis 1992; 1: 87-91.

3. Jamieson WR, Tyers GF, Janusz MT, et al. Age as a determinant for selection of porcine bioprostheses for cardiac valve replacement: experience with Carpentier-Edwards standard bioprosthesis. Can J Cardiol 1991; 7: 181-8.

4. Sanders SP, Levy RJ, Freed MD, Norwood WI, Castaneda AR. Use of Hancock porcine xenografts in children and adolescents. Am J Cardiol 1980; 46: 429-38.

5. Curcio CA, Commerford PJ, Rose AG, Stevens JE, Barnard MS. Calcification of glutaraldehyde-preserved porcine xenografts in young patients. J Thorac Cardiovasc Surg 1981; 81: 621-5.

6. Bortolotti U, Milano A, Mazzucco A, et al. Results of reoperation for primary tissue failure of porcine bioprostheses. J Thorac Cardiovasc Surg 1985; 90: 564-9.

7. Vahanian A, Baumgartner H, Bax J, et al. Guidelines on the management of valvular heart disease: The Task Force on the Management of Valvular Heart Disease of the European Society of Cardiology. Eur Heart J 2007; 28: 230-68.

8. Bortolotti U, Guerra F, Magni A, et al. Emergency reoperation for primary tissue failure of porcine bioprostheses. Am J Cardiol 1987; 60: 920-1. 
9. Bortolotti U, Milano A, Mossuto E, Mazzaro E, Thiene G, Casarotto D. Early and late outcome after reoperation for prosthetic valve dysfunction: analysis of 549 patients during a 26-year period. J Heart Valve Dis 1994; 3: 81-87.

10. Hammermeister K, Sethi GK, Henderson WG, Grover FL, Oprian C, Rahimtoola SH. Outcomes 15 years after valve replacement with a mechanical versus a bioprosthetic valve: final report of the Veterans Affairs randomized trial. J Am Coll Cardiol 2000; 36: 1152-8.

11. Khan SS, Trento A, DeRobertis M, et al. Twenty-year comparison of tissue and mechanical valve replacement. J Thorac Cardiovasc Surg 2001; 122: 257-6.

12. Mykén PS, Bech-Hansen O. A 20-year experience of 1712 patients with the Biocor porcine bioprosthesis. J Thorac Cardiovasc Surg 2009; 137: 76-81.

13. Rizzoli G, Mirone S, lus P, et al. Fifteen-year results with the Hancock II valve: a multicenter experience. J Thorac Cardiovasc Surg 2006; 132: 602-9.

14. David TE, Armstrong S, Maganti M. Hancock II bioprosthesis for aortic valve replacement: the gold standard of bioprosthetic valves durability? Ann Thorac Surg 2010; 90: 775-81.

15. McClure RS, Narayanasamy N, Wiegerinck E, et al. Late outcomes for aortic valve replacement with the Carpentier-Edwards pericardial bioprosthesis: up to 17-year follow-up in 1,000 patients. Ann Thorac Surg 2010; 89: 1410-6.

16. Chan V, Kulik A, Tran A, et al. Long-term clinical and hemodynamic performance of the Hancock II versus the Perimount aortic bioprostheses. Circulation 2010; 122 (11 Suppl): S10-6.

17. Dellgren G, David TE, Raanani E, Armstrong S, Ivanov J, Rakowski H. Late hemodynamic and clinical outcomes of aortic valve replacement with the Carpentier-Edwards Perimount pericardial bioprosthesis. J Thorac Cardiovasc Surg 2002; 124: 146-54.

18. Ruel M, Chan V, Bédard P, et al. Very long-term survival implications of heart valve replacement with tissue versus mechanical prostheses in adults $<60$ years ofage. Circulation 2007; 116 (11 Suppl): 1294-300.

19. Bortolotti U, Milano A, Mossuto E, et al. The risk of reoperation in patients with bioprosthetic valves. J Card Surg 1991; 6 (Suppl. 4): 638-43.

20. Stassano P, Di Tommaso L, Monaco M, et al. Aortic valve replacement: a prospective randomized evaluation of mechanical versus biological valves in patients ages 55 to 70 years. J Am Coll Cardiol 2009; 54: 1862-8.

21. Van Doorn CA, Stoodley KD, Saunders NR, Nair RU, Davies GA, Watson DA. Mitral valve replacement with the Carpentier-Edwards standard bioprosthesis: performance into the second decade. Eur J Cardiothorac Surg 1995; 9: 253-8.

22. Flameng $W$, Herregods MC, Vercalsteren $M$, Herijgers $P$, Bogaerts K, Meuris B. Prosthesis-patient mismatch predicts structural valve degeneration in bioprosthetic heart valves. Circulation 2010; 121: 2123-9.

23. Pelletier LC, Carrier M, Leclerc Y, Lepage G, deGuise P, Dyrda I. Porcine versus pericardial bioprostheses: a comparison of late results in 1,593 patients. Ann Thorac Surg 1989; 47: 352-61.

24. Pelletier LC, Carrier M, Leclerc Y, Dyrda I, Gosselin G. Influence of age on later results of valve replacement with porcine bioprostheses. J Cardiovasc Surg 1992; 33: 526-33.

25. Schoen FJ, Kujovich JL, Webb CL, Levy RJ. Chemically determined mineral content of explanted porcine aortic valve bioprostheses: correlation with radiographic assessment of calcification and clinical data. Circulation 1987; 76 : 1061-6.

26. Maciejewski M, Piestrzeniewicz K, Bielecka-Dąbrowa A, Piechowiak $M$, Jaszewski R. Redo surgery risk in patients with cardiac prosthetic valve dysfunction. Arch Med Sci 2011; 7: 271-7. 